\title{
NIL ALGEBRAS WITH NONRADICAL TENSOR SQUARE
}

\author{
EDMUND R. PUCZYLOWSKI
}

(Communicated by Donald S. Passman)

\begin{abstract}
The paper contains some simple observations on the tensor square of algebras. Applied to the well-known Golod examples, they allow us to produce nil algebras with nonradical tensor square.
\end{abstract}

In [1] Ananin constructed a finitely generated nil algebra $A$ over a field $K$ of characteristic zero, such that $A^{\omega}=\bigcap_{n=1}^{\infty} A^{n} \neq 0$ and $J\left(A \otimes_{K} A\right) \neq A \otimes_{K} A$, where $J$ denotes the Jacobson radical.

Here we make some simple observations which in particular show that examples of this sort can be constructed quite easily (of course on the basis of Golod's examples).

Throughout the note $K$ is a field. If it is not stated otherwise all considered algebras are $K$-algebras and tensor products are taken over $K$. The Jacobson radical of an algebra $A$ is denoted by $J(A)$.

LEMMA. If the field $K$ is ordered and for some $e_{1}, \ldots, e_{n}$ of a $K$-space $E$, $e_{1} \otimes e_{1}+\cdots+e_{n} \otimes e_{n}=0($ in $E \otimes E)$ then $e_{1}=e_{2}=\cdots=e_{n}=0$.

ProOF. Let $\left\{f_{i}\right\}$ be a $K$-basis of $E$ and $e_{j}=\sum a_{i j} f_{i}$, where $a_{i j} \in K$. Since

$$
0=e_{1} \otimes e_{1}+\cdots+e_{n} \otimes e_{n}=\sum_{k, l}\left(\sum_{i} a_{k i} a_{l i}\right)\left(f_{k} \otimes f_{l}\right)
$$

and $\left\{f_{k} \otimes f_{l}\right\}$ is a basis of $E \otimes E, \sum_{i} a_{k i} a_{l i}=0$ for all $k, l$. In particular $\sum_{i} a_{k i}^{2}=0$ for all $k$. This and the fact that the field $K$ is ordered give $a_{k i}=0$ for all $k, i$. Hence $e_{1}=e_{2}=\cdots=e_{n}=0$.

Proposition 1. If the field $K$ is ordered, $A$ is a $K$-algebra generated by $x_{1}, \ldots, x_{r}$ and $x_{1} \otimes x_{1}+\cdots+x_{r} \otimes x_{r}$ is a nilpotent element of $A \otimes A$ then the algebra $A$ is nilpotent.

PROOF. Obviously $A^{m}$ is generated by all possible products $e_{1}, \ldots, e_{t}$ of $m$ elements of the set $\left\{x_{1}, \ldots, x_{r}\right\}$ and $y=\left(x_{1} \otimes x_{1}+\cdots+x_{r} \otimes x_{r}\right)^{m}=e_{1} \otimes e_{1}+\cdots+$ $e_{t} \otimes e_{t}$. Hence if $y=0$ then by the lemma, $e_{1}=\cdots=e_{t}=0$ and $A^{m}=0$.

COROLlaRY 1. If the field $K$ is ordered then a $K$-algebra $A$ is locally nilpotent if and only if the algebra $A \otimes A$ is nil.

It is well known (cf. [3]) that if $\operatorname{dim}_{K} A<|K|$ then $J(A)$ is a nil ideal. Hence if $A$ is a finitely generated nonnilpotent algebra, the field $K$ is ordered and uncountable then $J(A \otimes A) \neq A \otimes A$. Algebras of this sort can be found among nil algebras

Received by the editors May 6, 1987.

1980 Mathematics Subject Classification (1985 Revision). Primary 16A22, 15A72. 
constructed by Golod in [2]. In fact all the algebras of [2] have in characteristic zero nonradical tensor square. Namely the construction gives that each such an algebra $A$ is isomorphic to $K \otimes_{Q} A^{\prime}$, for some algebra $A^{\prime}$ over the field $Q$ of rational numbers. Thus it suffices to show that if $B=A^{\prime} \otimes_{Q} A^{\prime}$ then $J(B) \neq B$. However the construction of $A$ gives also that $B=\bigoplus_{i=1}^{\infty} B_{i}$, where $B_{i}$ are $Q$-subspaces of $B$ with $B_{i} B_{j} \subseteq B_{i+j}$ ( $B$ is a graded algebra) and $B$ is generated by $B_{1}$. Now the result is an immediate consequence of Proposition 1 and the fact that elements of $J(B) \cap B_{1}$ are nilpotent. The latter can be proved as follows. There is an isomorphism from $B$ to the subalgebra $\sum_{i=1}^{\infty} B_{i} x^{i}$ of the polynomial algebra $B[x]$ which maps $b \in B_{1}$ onto $b x$. Now $f=b x+b^{2} x^{2}+\cdots$ is the quasi-inverse of $-b x$ in the power series ring $B[[x]]$. Hence if $b \in B_{1} \cap J(B)$ then $f \in B[x]$ and the element $b$ is nilpotent.

Thus the Golod construction gives in characteristic zero finitely generated nil algebras with nonradical tensor square. However these algebras, being graded, do not satisfy $A^{\omega} \neq 0$. Now we show how to modify them to obtain finitely generated nil algebras $A$ with $J(A \otimes A) \neq A \otimes A$ and $A^{\omega} \neq 0$. It is a consequence of the following more general result.

Proposition 2. For every algebra $A$ satisfying $J\left(A \otimes A^{0}\right) \neq A \otimes A^{0}$, where $A^{0}$ is the algebra opposite to $A$, there is an algebra $R$ with a nonzero ideal $I$ such that $R / I \approx A, I^{2}=0$ and $R^{\omega} \supseteq I$. In addition if the algebra $A$ is finitely generated then so is $R$.

ProOF. Let $A^{1}$ be the algebra obtained by an adjoining unity to $A$. Since $J\left(A \otimes A^{0}\right) \neq A \otimes A^{0}$, the subalgebra $B=A \otimes A^{0}+1 \otimes A^{0}+A \otimes 1$ of $A^{1} \otimes\left(A^{1}\right)^{0}$ is not radical. Hence there exists a simple left $B$-mociule $M$ with $B M=M$. Now putting for $a \in A$ and $m \in M, m \cdot a=(1 \otimes a) m$ and $a \cdot m=(a \otimes 1) m$ one obtains a structure of $A$-bimodule on $M$ (with the addition unchanged). The bimodule is simple and nondegenerated i.e. $A \cdot M \cdot A=M$. Now let $R$ be the set of matrices of the form $\left(\begin{array}{ll}a & m \\ 0 & a\end{array}\right)$, where $a \in A$ and $m \in M$. The set $R$ equipped with the componentwise addition and the multiplication given by

$$
\left(\begin{array}{cc}
a & m \\
0 & a
\end{array}\right)\left(\begin{array}{cc}
b & n \\
0 & b
\end{array}\right)=\left(\begin{array}{cc}
a b & a \cdot n+m \cdot b \\
0 & a b
\end{array}\right)
$$

is an algebra. Obviously

$$
I=\left\{\left(\begin{array}{cc}
0 & m \\
0 & 0
\end{array}\right) \mid m \in M\right\}
$$

is an ideal of $R$ with $I^{2}=0$ and $R / I \approx A$. It is easy to check, employing the fact that $M$ is a simple and nondegenerated $A$-bimodule, that $R^{\omega} \supseteq I$ and if the algebra $A$ is finitely generated then so is $R$.

The Golod examples of [2] give algebras with involution, so for those algebras $A \approx A^{0}$. Hence Proposition 2 can be applied to them, giving nil algebras $R$ with $J(R \otimes R) \neq R \otimes R$ and $R^{\omega} \neq 0$.

If $A$ is a nil algebra then so is the algebra $B=A \oplus A^{0}$. Obviously $B \approx B^{0}$ and if $J(A \otimes A) \neq A \otimes A$ then $J(B \otimes B) \neq B \otimes B$. This and Proposition 2 show that every nil algebra $A$ with $J(A \otimes A) \neq A \otimes A$ can be improved to a nil algebra $W$ with $J(R \otimes R) \neq R \otimes R$ and $R^{\omega} \neq 0$. 
ACKNOWLEDGMENT. I would liks to thank Z. Marciniak for a comment simplifying an earlier proof of the lriama.

\section{REFERENCES}

1. A. Z. Ananin, A nil algebra with nonradical tensor square, Sibirsk. Mat. Zh. 26 (1985), 192-194. (Russian)

2. E. S. Golod, On nil algebras and finitely approximable groups, Izv. Akad. Nauk SSSR Ser. Mat. 28 (1964), 273-276. (Russian)

3. N. Jacobson, Structure of rings, Amer. Math. Soc. Colloq. Publ., vol. 37, Amer. Math. Soc., Providence, R. I., 1956 (revised 1964).

Institute of Mathematics, University of Warsaw, PKin, 00-901 Warsaw, POLAND 